\title{
KHOVANOV HOMOLOGY OF THE 2-CABLE DETECTS THE UNKNOT
}

\author{
Matthew Hedden
}

\begin{abstract}
Inspired by recent work of Grigsby and Werhli, we use the deep geometric content of Ozsváth and Szabó's Floer homology theory to provide a short proof that the Khovanov homology of the 2-cable detects the unknot. A corollary is that Khovanov's categorification of the 2-colored Jones polynomial detects the unknot.
\end{abstract}

\section{Introduction}

A central problem in the study of quantum knot invariants is to determine if the Jones polynomial detects the unknot $[5,10]$. Even if it does not, one could hope that the Jones polynomial of cables of a knot might detect the unknot. To make this precise, recall that the $n$-cable of $K$, denoted $K^{n}$, is the $n$-component link obtained by taking $n$ parallel copies of $K$ (this operation depends on a framing, which we will always assume comes from a Seifert surface). Since isotopic knots have isotopic cables and the $n$-cable of the unknot, $U$, is the trivial $n$-component link, $U^{n}$, one could try to show that if $K$ is non-trivial then $J_{K^{n}} \neq J_{U^{n}}$ (here, $J$ denotes the Jones polynomial).

In [7], Khovanov introduced a link invariant which takes the form of a bi-graded homology theory. The graded Euler characteristic of his invariant is the Jones polynomial. Thus Khovanov's invariant contains as much information as the Jones polynomial, and one can relax the above questions to the context of Khovanov homology. The purpose of this note is to show that the Khovanov homology of the 2-cable detects the unknot.

Theorem 1.1. Let $K$ be a knot, and let $K^{2}$ denote its 2 -cable. Then $\mathrm{rk} K h\left(K^{2}\right)=4$ if and only if $K$ is the unknot. Here, $K h$ denotes the Khovanov homology (with coefficients in $\mathbb{Z} / 2 \mathbb{Z})$.

The key idea behind this theorem is that there is a spectral sequence from (reduced) Khovanov homology of $K^{2}$ to the Ozsváth-Szabó Floer homology of the branched double cover of $K^{2}$. The Thurston norm of this latter manifold is non-trivial in the case when $K$ is non-trivial. Since Ozsváth-Szabó Floer homology detects the Thurston norm, this forces the rank of Floer homology, and hence the rank of Khovanov homology, to grow.

We should remark that the Khovanov homology of cables is closely related to the so-called "colored" Khovanov invariants, whose graded Euler characteristics are the colored Jones polynomials (see [9] for definitions). The following is an immediate corollary of our proof.

Received by the editors August 25, 2008. 
Corollary 1.2. Khovanov's categorification of the 2-colored Jones polynomial detects the unknot.

The present work should be compared to that of Grigsby and Wehrli [3], who show that there are spectral sequences from Khovanov's categorification of the reduced colored Jones polynomial to an invariant coming from Juhasz's sutured Floer homology [6]. Though both results use spectral sequences from a Khovanov style invariant to a Floer invariant, the actual techniques are quite different.

It would also be interesting to compare our result with that of Andersen [2], who has announced a proof that the unknot is detected by the collection of Jones polynomials of all cables of a knot.

\section{Proof of Theorem}

All homology theories will have coefficients in $\mathbb{Z} / 2 \mathbb{Z}$.

Proof of Theorem 1.1 Assume that $K$ is a non-trivial knot.

Let $\Sigma(L)$ denote the double cover of $S^{3}$, branched along a link $L$. The proof begins by recalling Theorem 1.1 of [15], which says that there exists a spectral sequence whose $E_{2}$ term consists of Khovanov's reduced homology of the mirror of $L$, and which converges to the Ozsváth-Szabó "hat" Floer homology of $\Sigma(L)$. An immediate corollary (Corollary 1.2 of [15]) is that

$$
\text { rk } \widetilde{K h}(L) \geq \operatorname{rk} \widehat{H F}(\Sigma(L)) .
$$

Here, $\widetilde{K h}$ denotes the reduced Khovanov homology and $\widehat{H F}$ the "hat" Floer homology (see $[7,8]$ resp. [14] for relevant definitions).

Thus it remains to bound the rank of $\widehat{H F}\left(\Sigma\left(K^{2}\right)\right)$. To achieve this, we first observe that

$$
\Sigma\left(K^{2}\right) \cong S_{0}^{3}(K \# K),
$$

where $S_{0}^{3}(K \# K)$ denotes the manifold obtained by zero-framed surgery on the connected sum of $K$ with itself ${ }^{1}$. This can be seen, for instance, from the technique of Akbulut and Kirby [1] for finding a Kirby calculus description of the branched double cover of $L$ in terms of a Seifert surface. Specifically, apply the algorithm indicated by Figure 4 of [1] to an annulus bounded by $K^{2}$.

We now claim that if $K$ is non-trivial, then $\operatorname{rk} \widehat{H F}\left(S_{0}^{3}(K \# K)\right) \geq 6$. Essentially, this follows from the fact that the Thurston norm of this manifold is non-trivial, together with the fact that Floer homology detects the Thurston norm.

Let us be more precise. First, we have the elementary fact that the genus of $K \# K$ is $2 g$, where $g$ is the genus of $K$. Theorem 1.2 of [12] says that knot Floer homology detects the genus of $K$. In particular, it implies that $\widehat{H F K}(K \# K, 2 g) \neq 0$.

Now let $H F^{+}\left(S_{0}^{3}(K \# K), i\right)$ denote the "plus" Floer homology group associated to the $\operatorname{Spin}^{c}$ structure $\mathfrak{s}_{i} \in \operatorname{Spin}^{c}\left(S_{0}^{3}(K \# K)\right)$ whose Chern class satisfies $\left\langle c_{1}\left(\mathfrak{s}_{i}\right),[\widehat{F}]\right\rangle=$ $2 i$. Here, $[\widehat{F}] \in H_{2}\left(S_{0}^{3}(K \# K) ; \mathbb{Z}\right) \cong \mathbb{Z}$ is the generator obtained by capping off the oriented Seifert surface of $K \# K$ with the meridional disk of the surgery torus.

\footnotetext{
${ }^{1}$ Strictly speaking, we should reverse the string orientation of one of the copies of $K$ when taking the connected sum. Since our argument is insensitive to this discrepancy we suppress it from the notation.
} 
Since $2 g>1$, we can apply Corollary 4.5 of [11] to show that

$$
H F^{+}\left(S_{0}^{3}(K \# K), 2 g-1\right) \cong \widehat{H F K}(K \# K, 2 g) .
$$

An application of the long exact sequence relating $H F^{+}$to $\widehat{H F}$ (Lemma 4.4 of [14]) then shows that $\widehat{H F}\left(S_{0}^{3}(K \# K), 2 g-1\right) \neq 0$. Since the Euler characteristic of this group is zero (Proposition 5.1 of [13]), we have

$$
\text { rk } \widehat{H F}\left(S_{0}^{3}(K \# K), 2 g-1\right) \geq 2 .
$$

Floer homology enjoys a symmetry relation under the action of conjugation on Spin $^{c}$ structures (Theorem 2.4 of [13]). In the present context, this implies

$$
\text { rk } \widehat{H F}\left(S_{0}^{3}(K \# K),-2 g+1\right) \geq 2 .
$$

Finally, Theorem 10.1 of [13], together with the long exact sequence relating $\mathrm{HF}^{+}$, $H F^{-}$, and $H F^{\infty}$ shows that $H F^{+}\left(S_{0}^{3}(K \# K), 0\right) \neq 0$. By the same reasoning as above, we obtain

$$
\text { rk } \widehat{H F}\left(S_{0}^{3}(K \# K), 0\right) \geq 2,
$$

and have proved the claim.

Since the rank of the reduced Khovanov homology of the 2-component unlink is 2, we have shown that the reduced Khovanov homology of the two-cable detects the unknot. To obtain the statement in terms of the (unreduced) Khovanov homology, we observe that (since we are working with $\mathbb{Z} / 2 \mathbb{Z}$ coefficients),

$$
K h(K) \cong \widetilde{K h}(K) \otimes V,
$$

where $V$ is the rank 2 vector space obtained as the unreduced Khovanov homology of the unknot. This follows, for instance, from [16]. Thus, the Khovanov homology of the 2-cable of a non-trivial knot has rank at least 12.

Remark 2.1. The above argument extends in a straightforward manner to show that the Khovanov homology of the $2 n$-cable detects the unknot. A slight modification handles the case of odd cables. More precisely, the branched double cover of $K^{2 n+1}$ is obtained by gluing $F_{n} \times S^{1}$ to the (cyclic, unbranched) double cover of $S^{3} \backslash K$, where $F_{n}$ is a genus $n$ surface with a single boundary component. The gluing is performed by identifying $\{p t \in \partial F\} \times S^{1}$ with a longitude of $K$ (lifted to the cover), and $\partial F \times\{p t\}$ with the meridian. A homologically essential surface in $\Sigma\left(K^{2 n+1}\right)$ can be obtained by gluing $\gamma \times S^{1}$ to two oppositely oriented copies of a Seifert surface for $K$ (lifted to the cover), where $\gamma \in F_{n}$ is a properly embedded, homologically essential arc. Using this surface, we obtain lower bounds for the rank of Floer homology as before.

Proof of Corollary 1.2: From its definition [9], the rank of Khovanov's categorification of the 2-colored Jones polynomial differs from that of the Khovanov homology of the 2-cable by at most 1 . Thus the rank of Khovanov's categorification of the 2 -colored Jones polynomial of a non-trivial knot is at least 11, whereas it is 3 for the unknot. 


\section{Acknowledgements}

The inspiration for this note came from a talk I saw given by Stephan Wehrli at the "Knots in Washington XXVI" conference at George Washington University and from a conversation with Eli Grigsby at the 2008 AMS sectional meeting at LSU. I am indebted to them for their willingness to discuss their results. I also thank Liam Watson for interesting conversations, which led to [4]. This work was partially supported by NSF Grant DMS-0706979.

\section{References}

[1] S. Akbulut and R. Kirby, Branched covers of surfaces in 4-manifolds, Math. Ann. 252 (1980), no. $2,111-131$.

[2] J. Andersen. http://home.imf.au.dk/andersen/.

[3] J. E. Grigsby and S. Wehrli, On the Colored Jones Polynomial, Sutured Floer homology, and Knot Floer homology. Preprint, http://arxiv.org/abs/0807.1432.

[4] M. Hedden and L. Watson, Does Khovanov homology detect the unknot? To appear, Amer. J. Math. Preprint, http://arxiv.org/abs/0805.4423.

[5] V. F. R. Jones, A polynomial invariant for knots via von Neumann algebras, Bull. Amer. Math. Soc. (N.S.) 12 (1985), no. 1, 103-111.

[6] A. Juhász, Holomorphic discs and sutured manifolds, Algebr. Geom. Topol. 6 (2006) 1429-1457 (electronic).

[7] M. Khovanov, A categorification of the Jones polynomial, Duke Math. J. 101 (2000), no. 3, 359-426.

[8] - Patterns in knot cohomology. I, Experiment. Math. 12 (2003), no. 3, 365-374.

[9] —, Categorifications of the colored Jones polynomial, J. Knot Theory Ramifications 14 (2005), no. 1, 111-130.

[10] R. Kirby, Problems in low-dimensional topology, in Geometric topology (Athens, GA, 1993),, Vol. 2 of AMS/IP Stud. Adv. Math., Amer. Math. Soc. (1997).

[11] P. Ozsváth and Z. Szabó, Holomorphic disks and knot invariants, Adv. Math. 186 (2004), no. 1, $58-116$.

[12] P. S. Ozsváth and Z. Szabó, Holomorphic Disks and Genus Bounds, Geom. Topol. 8 (2004) 311-334.

[13] - Holomorphic disks and three-manifold invariants: properties and applications, Ann. of Math. 159 (2004), no. 3, 1159-1245.

[14] - Holomorphic disks and topological invariants for closed three-manifolds, Ann. of Math. (2) 159 (2004), no. 3, 1027-1158.

[15] - On the Heegaard Floer homology of branched double-covers, Adv. Math. 194 (2005), no. $1,1-33$.

[16] A. Shumakovitch, Torsion of the Khovanov homology. Preprint, available at http://arxiv.org/abs/math/0405474.

Mathematics Department, Michigan State University, East Lansing, Mi 48824

E-mail address: mhedden@math.msu.edu 